\title{
The role of ferroptosis in digestive system cancer (Review)
}

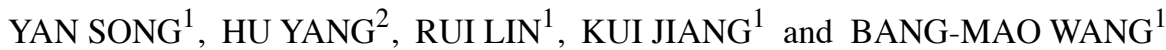 \\ ${ }^{1}$ Department of Gastroenterology, Tianjin Medical University General Hospital, Tianjin 300052; \\ ${ }^{2}$ Department of Nephrology, Second Hospital of Tianjin Medical University, Tianjin 300211, P.R. China
}

Received December 20, 2018; Accepted June 11, 2019

DOI: $10.3892 / \mathrm{ol} .2019 .10568$

\begin{abstract}
Ferroptosis is a type of regulated cell death dependent on iron and reactive oxygen species. Ferroptosis is distinct from other cell death modalities, including apoptosis, autophagy and necrosis. Dysregulated ferroptosis has been implicated in a number of diseases, including neuropathy, ischemia reperfusion injury, acute kidney failure and cancer. The digestive system consists of several organs. The morbidity and mortality rates of digestive system cancer are high. The current review summarizes the role of ferroptosis in digestive system cancer. A large number of molecules, including tumor protein p53, retinoblastoma protein, nuclear factor E2-related factor 2, KH RNA binding domain containing signal transduction associated 1 , cysteine dioxygenase type 1 , metallothionein-1G, nuclear receptor coactivator $4, \mathrm{CDGSH}$ iron sulfur domain 1 , heat shock protein family A (Hsp70) member 5 and acyl-CoA synthetase long chain family member 4 , regulate ferroptosis in digestive system cancer. Drugs such as cisplatin, baicalein, haloperidol, artesunate, piperlongumine, saponin and bromelain may cause cancer cell death by inducing ferroptosis. An improved understanding of ferroptosis in digestive system cancer may give rise to novel diagnostic and making therapeutic strategies.
\end{abstract}

\section{Contents}

1. Introduction

2. Ferroptosis in digestive system cancer

3. The association between ferroptosis and other forms of regulated cell death

4. Conclusions

Correspondence to: Dr Yan Song, Department of Gastroenterology, Tianjin Medical University General Hospital, 154 Anshan Road, Tianjin 300052, P.R. China

E-mail: somebodyaaa@126.com

Key words: ferroptosis, regulated cell death, digestive cancer

\section{Introduction}

Ferroptosis is a novel form of regulated cell death (RCD) that was described in 2012 (1). Ferroptosis involves the accumulation of lipid peroxidation products and reactive oxygen species (ROS) derived from iron metabolism. Ferroptosis is morphologically, biochemically and genetically distinct from other pathways for RCD, including necroptosis, apoptosis and autophagy (2). Amino acid, iron and lipid metabolism are involved in the process of ferroptosis. Glutamate and glutamine are pivotal regulators of ferroptosis (3). During normal physiological function, extracellular glutamate induces ferroptosis. Lipid metabolism may affect the sensitivity of cells to ferroptosis. Polyunsaturated fatty acids are susceptible to lipid peroxidation and are required for ferroptosis (4). Iron is involved in the accumulation of lipid peroxides and ferroptosis. Iron import, export, storage and turnover influence the sensitivity of cells to ferroptosis (5). Several molecules, including voltage dependent anion channel (VDAC) $2 / 3$, glutathione peroxidase 4 (GPX4), heat shock protein $\beta-1$, nuclear factor E2-related factor 2 (NRF2), NADPH oxidase, the tumor suppressor p53 (TP53) and solute carrier family 7 member 1 (SLC7A1), regulate ferroptosis through the direct or indirect targets of iron metabolism as well as lipid peroxidation (2).

Previous studies demonstrated that ferroptosis participated in diverse diseases, including neuropathy $(1,6)$, ischemia reperfusion injury (3) acute kidney failure (6-8) and cancer (3). Sorafenib was revealed to induce ferroptosis in hepatocellular carcinoma (HCC) and several studies have subsequently investigated the mechanism of ferroptosis in HCC (9-11). The digestive system comprises several organs and the incidence of cancer in the digestive system is high. The current review presents the role of ferroptosis in digestive system cancer.

\section{Ferroptosis in digestive system cancer}

Ferroptosis was revealed to be involved in various types of digestive system cancer, excluding esophageal and biliary system cancer $(9,12-15)$. The mechanisms of ferroptosis in digestive system cancer are presented in Table I and Fig. 1.

Gastric cancer. Studies investigating ferroptosis in gastric cancer are lacking. Human cysteine dioxygenase 1 (CDO1) converts cysteine to taurine (16); therefore, an increase in CDO1 activity is expected to decrease cellular cysteine levels. Cysteine is required for glutathione (GSH) synthesis 
and increased CDO1 activity may decrease the synthesis of GSH and promote ferroptosis (16). Hao et al (12) revealed that that silencing of CDO1 in gastric cancer cells reduced the erastin-induced ferroptosis by restoring cellular GSH, which prevented ROS generation and lipid peroxidation. MYB proto-oncogene transcription factor, which interacts with the CDO1 promoter as a transcription factor, regulated CDO1 and GPX4 expression during ferroptosis (12). Further investigation is required to identify the role and underlying mechanism of ferroptosis in gastric cancer.

Colon cancer. Previous studies investigating the role of ferroptosis in colon cancer have largely focused on TP53 (13,17-19). TP53 is an evolutionarily conserved protein involved in the regulation of cell proliferation, death and differentiation and metabolism. TP53 may initiate cell-cycle arrest and induce apoptosis in response to stress stimuli by means of the transcriptional and transcription-independent processes (17). Additionally, the induction of ferroptosis may contribute to the oncosuppressive activities of TP53 (13). Furthermore, TP53 was revealed to promote ferroptosis by means of a transcription-dependent process (20). Xie et al (20) revealed that TP53 constrained erastin-stimulated ferroptosis through the inhibition of dipeptidyl-peptidase-4 (DPP4) function in a transcription-independent manner in colorectal cancer. Silencing of TP53 facilitated plasma membrane-associated DPP4-dependent lipid peroxidation, and thus prevented nuclear accumulation of DPP4. As a result, SLC7A11 expression was increased which resulted in ferroptosis (20). Furthermore, the TP53-independent p53 upregulated modulator of apoptosis axis was implicated in ferroptosis induction in human colon cancer HCT116 cells (19).

The role of TP53 in ferroptosis may provide a therapeutic target for colon cancer. Albiziabioside A, a saponin derivative, suppressed the expression of GPX4, and together with the accumulation of lipid peroxidation products, resulted in ferroptosis by TP53 activation (21). Cisplatin induced ferroptosis by depleting GSH and inactivating GPX4 in A549 and HCT116 cells, suggesting that ferroptosis may be a novel target in cancer therapy (22). Bromelain exhibited anticancer effects in Kras mutant human colorectal carcinoma cells by upregulating the expression of acyl-CoA synthetase long chain family member 4, which acted as a ferroptosis target (23).

Defective ribosome biogenesis was reported in colon cancer lacking TP53 (24). 5 -fluorouracil induced ribosomal protein L3 (RPL3) activation in TP53-/- colon cancer cells by inhibiting the expression of cystathionine- $\beta$-synthase (24), which may subsequently influence the cysteine level. Additionally, RPL3 decreased the expression of cysteine/glutamate antiporter and induced ferroptosis in TP53-mutated lung cancer cells (25). Ribosomal protein-dependent pathways are associated with ferroptosis and may be potential targets of anticancer drugs.

Pancreatic cancer. Pancreatic cancer is one of the most lethal types of digestive malignancies, and current chemotherapeutic drugs are often ineffective. Previous studies suggested that certain natural plant extracts possess a potential therapeutic effect in pancreatic cancer cells by inducing ferroptosis $(15,18,26,27)$. The combination of cotylenin A (CN-A) and phenethyl isothiocyanate synergistically induced pancreatic cancer cell death by the generation of ROS, which drives ferroptosis (26). In addition, artesunate (ART) induced ferroptosis by ROS generation and lipid peroxidation in KRAS-activated human pancreatic ductal adenocarcinoma (PDAC) and the AT-induced ferroptosis was inhibited by ferrostatin-1. Notably, ART did not exert an effect on non-neoplastic human pancreatic ductal epithelial cells (27). This suggests that ART may be a candidate for pancreatic cancer therapy by inducing ferroptosis. Piperlongumine (PL) induces human pancreatic cancer cell line death by substantially increasing the intracellular ROS level. This effect was inhibited by ferroptosis inhibitors and iron chelators but not apoptosis or necrosis inhibitors, suggesting that PL induced cell death by ferroptosis (15). Furthermore, the aforementioned study revealed that triple combined therapy with PL, CN-A and sulfasalazine had a high efficacy in pancreatic cancer in vitro (15). Baicalein inhibited erastin-induced ferroptosis by preventing GSH depletion and GPX4 degradation in pancreatic cancer cells (18).

Previous studies have investigated the mechanism of ferroptosis in pancreatic cancer cells $(14,28,29)$. Heat shock protein family A (Hsp70) member 5 (HSPA5) negatively regulated ferroptosis in human PDAC cells through the HSPA5-GPX4 signaling pathway, which mediated ferroptosis resistance (14). The inhibition of the HSPA5-GPX4 signaling pathway improved gemcitabine sensitivity through the disinhibition of ferroptosis in vitro and in vivo (14). Nuclear receptor coactivator 4 (NCOA4) was revealed as the cargo receptor mediating ferroptosis (28). Silencing of NCOA4 inhibited ferritin degradation and suppressed ferroptosis while the overexpression of NCOA4 augmented ferritin degradation and promoted ferroptosis in human pancreatic cells (30). Lipoxygenase inhibitors prevented pancreatic cell ferroptosis induced by erastin as well as the ferroptosis inhibitor RSL3. In addition, treatment of the human pancreatic cancer cell line Panc-1 with arachidonate 15-lipoxygenase-activating compounds accelerated ferroptosis (29). The TP53-independent PUMA axis was implicated in ferroptosis in human pancreatic cancer cells as well as the previously mentioned colon cancer cells (19).

$H C C$. The majority of previous studies associated with ferroptosis involved the investigation of $\operatorname{HCC}(10,31,32)$. Sorafenib, a multikinase inhibitor, is used for the treatment of advanced HCC and is currently being investigated as a ferroptosis inducer $(2,9)$. Mechanistically, sorafenib induces ferroptosis as a form of regulated cell death in HCC. Sorafenib inhibits the initiation of translation mediated by the rapamycin kinase signaling pathway which is considered to constitutes an important role in the initiation of ferroptosis in HCC (11). Iron metabolism is identified as a vital mediator of ferroptosis and the iron chelator deferoxamine prevents sorafenib from inducing oxidative stress in HCC cells (31). CDGSH iron sulfur domain 1 (CISD1) is an iron-possessing external mitochondrial membrane protein that modulates mitochondrial iron uptake and respiration (33). A previous study revealed that CISD1 decreased ferroptotic cell death in HCC cells by regulating iron metabolism and preventing mitochondrial damage in ferroptosis (32). In addition to iron metabolism regulating ferroptosis, lipid metabolism may serve an important role in the regulation 


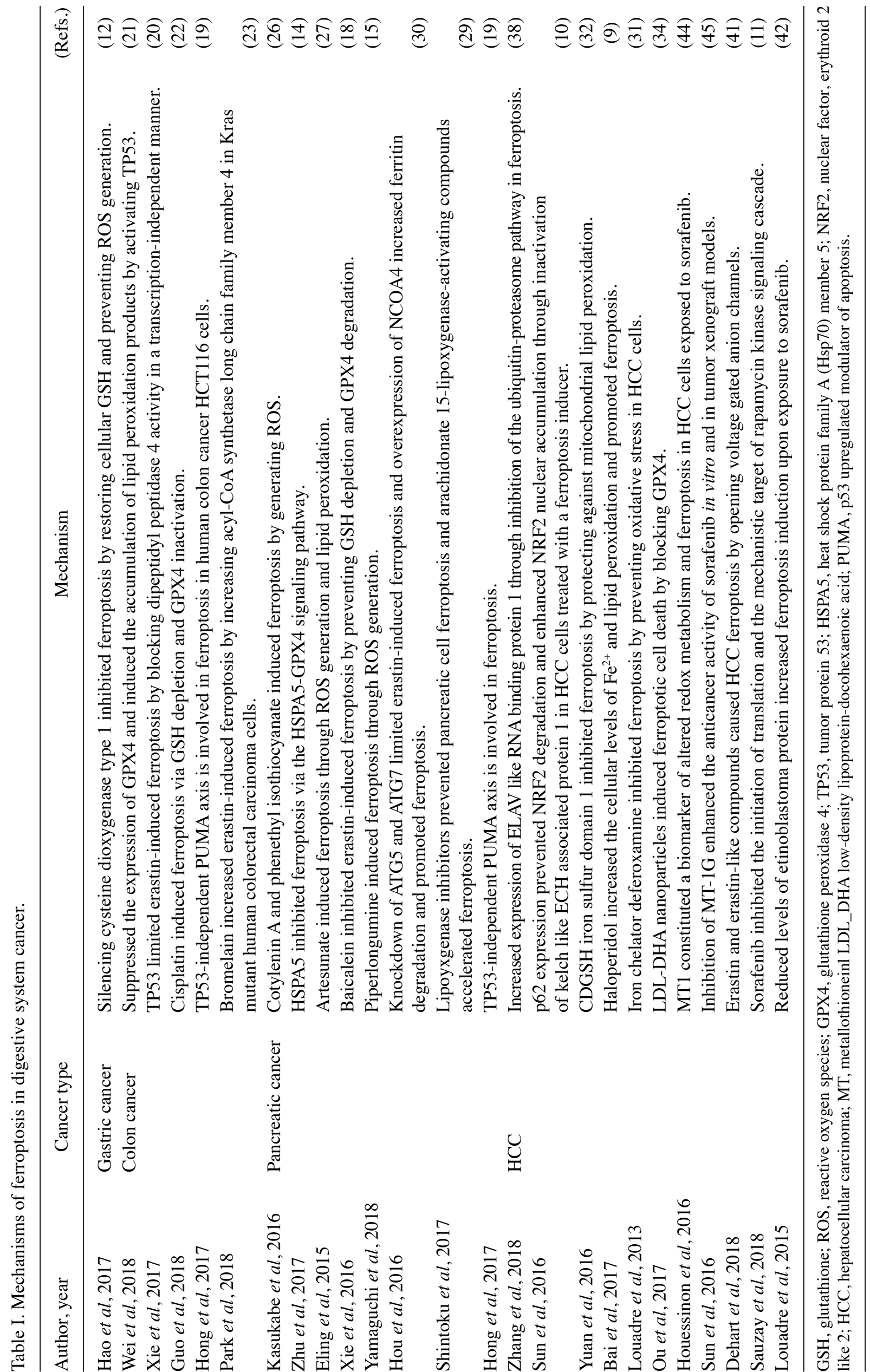




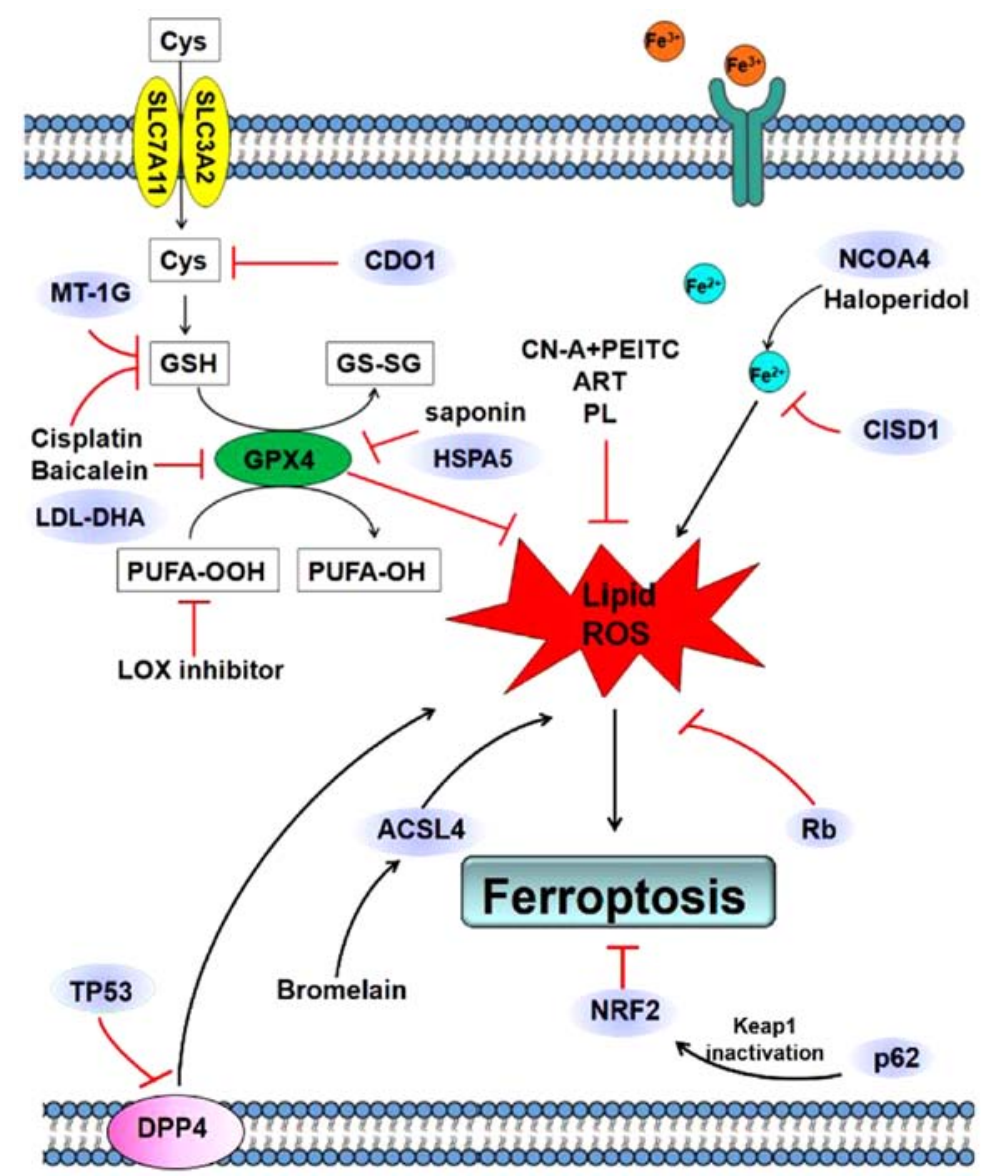

Figure 1. Signaling pathways regulating ferroptosis in cancer of the digestive system.

of ferroptosis (31). Low-density lipoproteins-docosahexaenoic acid nanoparticles stimulated ferroptosis by promoting lipid peroxidation, depleting glutathione and inactivating the lipid antioxidant GPX4 in a rat hepatoma model, HCC cell lines and human HCC tumor xenografts in mice (34).

NRF2 is implicated in several types of cancer, including HCC (35-37). RF2 is involved in ferroptosis in HCC cells. When $\mathrm{HCC}$ cells were exposed to a ferroptosis inducer, p62 expression decreased NRF2 degradation and increased NRF2 nuclear accumulation by inactivating Kelchlike ECH-associated protein 1 (KEAP1). Knockdown of p62 in HCC cells promoted the anticancer function of erastin and sorafenib by inducing ferroptosis (10). Activating the p62-KEAP1-NRF2 signaling pathway may thus protect against ferroptosis in HCC cells (10). Furthermore, NRF2 determined the therapeutic efficacy of ferroptosis-targeted therapies in HCC cells (10).

Zhang et al (38) revealed that ELAV-like RNA binding protein 1 (ELAVL1) serves an important role in the regulation of ferroptosis in liver fibrosis and hepatocarcinoma. When erastin was exposed to ferroptosis-inducing compounds, ELAVL1 protein expression was significantly increased due to inhibition of the ubiquitin-proteasome pathway (38). The $\sigma 1$ receptor $(\mathrm{S} 1 \mathrm{R})$ is present in the mitochondrial membrane and endoplasmic reticulum and modulates oxidative stress $(39,40)$. Haloperidol, an S1R antagonist, significantly increased the cellular concentration of $\mathrm{Fe}^{2+}$ as well as lipid peroxidation, and accordingly promoted erastin-and sorafenib-induced ferroptosis (9). Furthermore, erastin, coupled with other erastin-like lead compounds, induced HCC ferroptosis through the opening of VDACs, which increased the membrane potential, mitochondrial ROS and oxidative stress-induced cell death (41).

Louandre et al (42) revealed that HCC cells with decreased expression of retinoblastoma protein $(\mathrm{Rb})$ manifested a 2-to 3 -fold increase in ferroptotic cell death induced by sorafenib compared with controls, highlighting the function of $\mathrm{Rb}$ in determining the susceptibility of HCC cells to sorafenib as well as regulating ferroptosis. However, clinically-applicable biomarkers reflecting the susceptibility of cancer cells to sorafenib are still in shortage (43). Microarray analysis and subsequent experiments revealed that metallothionein (MT)-1 may be a biomarker of modified redox metabolism as well as ferroptosis in HCC cells exposed to sorafenib (44). Furthermore, MT-1G may serve as a potential therapeutic target for overcoming sorafenib resistance in human HCC cells (45). Additionally, knockdown of MT-1G by RNA interference augmented glutathione depletion and lipid peroxidation, which promoted sorafenib-induced ferroptosis (45).

\section{The association between ferroptosis and other forms of regulated cell death}

While ferroptosis is distinct from other forms of regulated cell death, including apoptosis, necrosis and autophagy, it is connected with other types of cell deaths $(19,30)$. 
Apoptosis. ART and erastin, which are common ferroptosis inducers, enhance tumor necrosis factor-related apoptosis-inducing ligand (TRAIL)-induced apoptosis in human pancreatic cancer PANC-1 and BxPC-3 cells as well as human colon cancer HCT116 cells (19). An increased activation of caspase $8 / 9 / 3$, regarded as the hallmark of apoptosis, was observed in the aforementioned study. In vitro, the ferroptotic agents stimulated endoplasmic reticulum (ER) stress in human colon cells (46). Furthermore, they increased the activation of the death receptor 5 via the TRAIL and initiated apoptosis (46).

Autophagy. A recent study revealed that autophagy was conducive to ferroptosis by degrading ferritin in cancer cells. Knockdown of autophagy-related (ATG) 5 and ATG7 limited erastin-stimulated ferroptosis by reducing the intracellular ferrous iron concentration and lipid peroxidation (30). ELAVL1 is a positive regulator of ferroptosis by inhibiting the ubiquitin-proteasome pathway. Upregulated ELAVL1 expression revealed increased autophagosome production coupled with autophagic flux. ELAVL1 promoted autophagy by binding to the adenine uracil-rich region in the 3'-untranslated region of beclin 1 (BECN1) mRNA, resulting in BECN1 overexpression (38).

ER stress. Previous studies have revealed that inhibiting cystine-glutamate exchange with the aid of ferroptotic agents activates an ER stress feedback loop and upregulates the glutathione-specific $\gamma$ glutamylcyclotransferase 1 gene $(47,48)$. Hong et al (14) demonstrated that the unfolded protein response was detected in ART-treated colon cancer cells, suggesting that the ferroptotic agent induced ER stress. Furthermore, the ER stress markers HSPA5 and DNA damage inducible transcript 3 were detected in ferroptotic agent-treated HCT116 cells, suggesting that ferroptotic agents induce ER stress $(14,19)$. Additionally, ART-induced ER stress was inhibited by iron chelators and lipid peroxidation inhibitors, including ferrostatin-1 and lipoxstatin-1.

\section{Conclusions}

The digestive system consists of a number of organs and cancer of the digestive system has a relatively high morbidity and mortality rates. Furthermore, patients with digestive system cancer have a poor quality of life. Ferroptosis, which is a novel form of regulated cell death, is implicated in several types of digestive system cancer, excluding esophageal and biliary system cancer. As described in the present review, iron, lipid and amino acid metabolism are involved in ferroptosis. Molecules involved in ferroptosis in digestive system cancer, include TP53, Rb, NRF2, p62, CDO1, MT-1G, NCOA4, CISD1 and HSPA5. Several drugs, such as cisplatin, baicalein, haloperidol, ART, PL, bromelain and saponin, induce cancer cell death by ferroptosis in the digestive system and exert therapeutic effects. Nevertheless, the signaling pathways and major transcriptional regulators of ferroptosis in digestive system cancer remain unknown. Further studies are required to establish the roles of ferroptosis in metastasis, energy metabolism, autophagy and drug resistance. Furthermore, the elucidation of molecular pathways underlying ferroptosis in digestive system cancer may provide novel therapeutic targets and improve the prognosis of patients with digestive system cancer.

\section{Acknowledgements}

Not applicable.

\section{Funding}

The present review was supported by the National Natural Science Foundation of China (grant no. 81600509) and the Youth Incubation foundation of Tianjin Medical University General Hospital (grant no. ZYYFY2016020).

\section{Availability of data and materials}

Not applicable.

\section{Authors' contributions}

YS and RL conceived and designed the review. YS and HY drafted the manuscript. KJ and BMW critically revised the article for intellectual content. All the authors approved the final version of manuscript.

\section{Ethics approval and consent to participate}

Not applicable.

\section{Patient consent for publication}

Not applicable.

\section{Competing interests}

Not applicable.

\section{References}

1. Dixon SJ, Lemberg KM, Lamprecht MR, Skouta R, Zaitsev EM, Gleason CE, Patel DN, Bauer AJ, Cantley AM, Yang WS, et al: Ferroptosis: An iron-dependent form of nonapoptotic cell death. Cell 149: 1060-1072, 2012.

2. Xie Y, Hou W, Song X, Yu Y, Huang J, Sun X, Kang R and Tang D: Ferroptosis: Process and function. Cell death Differ 23: 369-379, 2016.

3. Gao M, Monian P, Quadri N, Ramasamy R and Jiang X: Glutaminolysis and transferrin regulate ferroptosis. Mol Cell 59: 298-308, 2015.

4. Yang WS and Stockwell BR: Ferroptosis: Death by lipid peroxidation. Trends Cell Biol 26: 165-176, 2016.

5. Stockwell BR, Friedmann Angeli JP, Bayir H, Bush AI, Conrad M, Dixon SJ, Fulda S, Gascón S, Hatzios SK, Kagan VE, et al: Ferroptosis: A regulated cell death nexus linking metabolism, redox biology and disease. Cell 171: 273-285, 2017.

6. Skouta R, Dixon SJ, Wang J, Dunn DE, Orman M, Shimada K, Rosenberg PA, Lo DC, Weinberg JM, Linkermann A and Stockwell BR: Ferrostatins inhibit oxidative lipid damage and cell death in diverse disease models. J Am Chem Soc 136: 4551-4556, 2014

7. Friedmann Angeli JP, Schneider M, Proneth B, Tyurina YY, Tyurin VA, Hammond VJ, Herbach N, Aichler M, Walch A, Eggenhofer E, et al: Inactivation of the ferroptosis regulator Gpx4 triggers acute renal failure in mice. Nat Cell Biol 16: 1180-1191, 2014. 
8. Linkermann A, Skouta R, Himmerkus N, Mulay SR, Dewitz C, De Zen F, Prokai A, Zuchtriegel G, Krombach F, Welz PS, et al: Synchronized renal tubular cell death involves ferroptosis. Proc Natl Acad Sci USA 111: 16836-16841, 2014.

9. Bai T, Wang S, Zhao Y, Zhu R, Wang W and Sun Y: Haloperidol, a sigma receptor 1 antagonist, promotes ferroptosis in hepatocellular carcinoma cells. Biochem Biophys Res Commun 491: 919-925, 2017.

10. Sun X, Ou Z, Chen R, Niu X, Chen D, Kang R and Tang D: Activation of the p62-Keap1-NRF2 pathway protects against ferroptosis in hepatocellular carcinoma cells. Hepatology 63: 173-184, 2016.

11. Sauzay C, Louandre C, Bodeau S, Anglade F, Godin C, Saidak Z, Fontaine JX, Usureau C, Martin N, Molinie R, et al: Protein biosynthesis, a target of sorafenib, interferes with the unfolded protein response (UPR) and ferroptosis in hepatocellular carcinoma cells. Oncotarget 9: 8400-8414, 2018.

12. Hao S, Yu J, He W, Huang Q, Zhao Y, Liang B, Zhang S, Wen Z, Dong S, Rao J, et al: Cysteine dioxygenase 1 mediates erastin-induced ferroptosis in human gastric cancer cells. Neoplasia 19: 1022-1032, 2017.

13. Jennis M, Kung CP, Basu S, Budina-Kolomets A, Leu JI, Khaku S, Scott JP, Cai KQ, Campbell MR, Porter DK, et al: An african-specific polymorphism in the TP53 gene impairs p53 tumor suppressor function in a mouse model. Genes Dev 30 : 918-930, 2016.

14. Zhu S, Zhang Q, Sun X, Zeh HJ III, Lotze MT, Kang R and Tang D: HSPA5 regulates ferroptotic cell death in cancer cells. Cancer Res 77: 2064-2077, 2017

15. Yamaguchi Y, Kasukabe T and Kumakura S: Piperlongumine rapidly induces the death of human pancreatic cancer cells mainly through the induction of ferroptosis. Int $\mathrm{J}$ Oncol 52 : 1011-1022, 2018

16. Stipanuk MH, Ueki I, Dominy JE Jr, Simmons CR and Hirschberger LL: Cysteine dioxygenase: A robust system for regulation of cellular cysteine levels. Amino acids 37: 55-63, 2009.

17. Khoo KH, Verma CS and Lane DP: Drugging the p53 pathway: Understanding the route to clinical efficacy. Nat Rev Drug Discov 13: 217-236, 2014

18. Xie Y, Song X, Sun X, Huang J, Zhong M, Lotze MT, Zeh HJ Rd, Kang $\mathrm{R}$ and Tang D: Identification of baicalein as a ferroptosis inhibitor by natural product library screening. Biochem Biophys Res Commun 473: 775-780, 2016.

19. Hong SH, Lee DH, Lee YS, Jo MJ, Jeong YA, Kwon WT, Choudry HA, Bartlett DL and Lee YJ: Molecular crosstalk between ferroptosis and apoptosis: Emerging role of ER stress-induced p53-independent PUMA expression. Oncotarget 8 : 115164-115178, 2017.

20. Xie Y, Zhu S, Song X, Sun X, Fan Y, Liu J, Zhong M, Yuan H, Zhang L, Billiar TR, et al: The tumor suppressor p53 limits ferroptosis by blocking DPP4 activity. Cell Rep 20: 1692-1704, 2017.

21. Wei G, Sun J, Hou Z, Luan W, Wang S, Cui S, Cheng M and Liu Y: Novel antitumor compound optimized from natural saponin albiziabioside a induced caspase-dependent apoptosis and ferroptosis as a p53 activator through the mitochondrial pathway. Eur J Med Chem 157: 759-772, 2018.

22. Guo J, Xu B, Han Q, Zhou H, Xia Y, Gong C, Dai X, Li Z and Wu G: Ferroptosis: A novel anti-tumor action for cisplatin. Cancer Res Treat 50: 445-460, 2018.

23. Park S, Oh J, Kim M and Jin EJ: Bromelain effectively suppresses Kras-mutant colorectal cancer by stimulating ferroptosis. Anim Cells Syst (Seoul) 22: 334-340, 2018.

24. Pagliara V, Saide A, Mitidieri E, d'Emmanuele di Villa Bianca R, Sorrentino R, Russo G and Russo A: 5-FU targets rpL3 to induce mitochondrial apoptosis via cystathionine- $\beta$-synthase in colon cancer cells lacking p53. Oncotarget 7: 50333-50348, 2016.

25. Russo A, Saide A, Smaldone S, Faraonio R and Russo G: Role of uL3 in multidrug resistance in p53-mutated lung cancer cells. Int J Mol Sci 18: pii: E547, 2017.

26. Kasukabe T, Honma Y, Okabe-Kado J, Higuchi Y, Kato N and Kumakura S: Combined treatment with cotylenin A and phenethyl isothiocyanate induces strong antitumor activity mainly through the induction of ferroptotic cell death in human pancreatic cancer cells. Oncol Rep 36: 968-976, 2016.

27. Eling N, Reuter L, Hazin J, Hamacher-Brady A and Brady NR: Identification of artesunate as a specific activator of ferroptosis in pancreatic cancer cells. Oncoscience 2: 517-532, 2015.
28. Mancias JD, Wang X, Gygi SP, Harper JW and Kimmelman AC: Quantitative proteomics identifies NCOA4 as the cargo receptor mediating ferritinophagy. Nature 509: 105-109, 2014.

29. Shintoku R, Takigawa Y, Yamada K, Kubota C, Yoshimoto Y, Takeuchi T, Koshiishi I and Torii S: Lipoxygenase-mediated generation of lipid peroxides enhances ferroptosis induced by erastin and RSL3. Cancer Sci 108: 2187-2194, 2017.

30. Hou W, Xie Y, Song X, Sun X, Lotze MT, Zeh HJ III, Kang R and Tang D: Autophagy promotes ferroptosis by degradation of ferritin. Autophagy 12: 1425-1428, 2016.

31. Louandre C, Ezzoukhry Z, Godin C, Barbare JC, Mazière JC, Chauffert B and Galmiche A: Iron-dependent cell death of hepatocellular carcinoma cells exposed to sorafenib. Int J Cancer 133: 1732-1742, 2013.

32. Yuan H, Li X, Zhang X, Kang R and Tang D: CISD1 inhibits ferroptosis by protection against mitochondrial lipid peroxidation. Biochem Biophys Res Commun 478: 838-844, 2016.

33. Geldenhuys WJ, Leeper TC and Carroll RT: mitoNEET as a novel drug target for mitochondrial dysfunction. Drug Discov Today 19: 1601-1606, 2014.

34. Ou W, Mulik RS, Anwar A, McDonald JG, He X and Corbin IR: Low-density lipoprotein docosahexaenoic acid nanoparticles induce ferroptotic cell death in hepatocellular carcinoma. Free Radic Biol Med 112: 597-607, 2017.

35. Sporn MB and Liby KT: NRF2 and cancer: The good, the bad and the importance of context. Nat Rev Cancer 12: 564-571, 2012.

36. Jaramillo MC and Zhang DD: The emerging role of the Nrf2-Keap1 signaling pathway in cancer. Genes Dev 27: 2179-2191, 2013.

37. Ren D, Villeneuve NF, Jiang T, Wu T, Lau A, Toppin HA and Zhang DD: Brusatol enhances the efficacy of chemotherapy by inhibiting the Nrf2-mediated defense mechanism. Proc Natl Acad Sci USA 108: 1433-1438, 2011.

38. Zhang Z, Yao Z, Wang L, Ding H, Shao J, Chen A, Zhang F and Zheng S: Activation of ferritinophagy is required for the RNA-binding protein ELAVL1/HuR to regulate ferroptosis in hepatic stellate cells. Autophagy 14: 2083-2103, 2018.

39. Hayashi T and Su TP: Sigma-1 receptor chaperones at the ER-mitochondrion interface regulate $\mathrm{Ca}(2+)$ signaling and cell survival. Cell 131: 596-610, 2007.

40. Kourrich S, Hayashi T, Chuang JY, Tsai SY, Su TP and Bonci A: Dynamic interaction between sigma-1 receptor and Kv1.2 shapes neuronal and behavioral responses to cocaine. Cell 152: 236-247, 2013.

41. Dehart DN, Fang D, Heslop K, Li L, Lemasters JJ and Maldonado EN: Opening of voltage dependent anion channels promotes reactive oxygen species generation, mitochondrial dysfunction and cell death in cancer cells. Biochem Pharmacol 148: 155-162, 2018

42. Louandre C, Marcq I, Bouhlal H, Lachaier E, Godin C, Saidak Z, François C, Chatelain D, Debuysscher V, Barbare JC, et al: The retinoblastoma $(\mathrm{Rb})$ protein regulates ferroptosis induced by sorafenib in human hepatocellular carcinoma cells. Cancer Lett 356: 971-977, 2015.

43. Cao W, Hou FF and Nie J: AOPPs and the progression of kidney disease. Kidney Int Suppl (2011) 4: 102-106, 2014.

44. Houessinon A, François C, Sauzay C, Louandre C, Mongelard G Godin C, Bodeau S, Takahashi S, Saidak Z, Gutierrez L, et al: Metallothionein-1 as a biomarker of altered redox metabolism in hepatocellular carcinoma cells exposed to sorafenib. Mol Cancer 15: 38, 2016.

45. Sun X, Niu X, Chen R, He W, Chen D, Kang R and Tang D: Metallothionein-1G facilitates sorafenib resistance through inhibition of ferroptosis. Hepatology 64: 488-500, 2016.

46. Lee YS, Lee DH, Jeong SY, Park SH, Oh SC, Park YS, Yu J, Choudry HA, Bartlett DL and Lee YJ: Ferroptosis-inducing agents enhance TRAIL-induced apoptosis through upregulation of death receptor 5. J Cell Biochem 120: 928-939, 2019.

47. Dixon SJ, Patel DN, Welsch M, Skouta R, Lee ED, Hayano M, Thomas AG, Gleason CE, Tatonetti NP, Slusher BS and Stockwell BR: Pharmacological inhibition of cystine-glutamate exchange induces endoplasmic reticulum stress and ferroptosis. Elife 3: $\mathrm{e} 02523,2014$.

48. Dai Z, Huang Y, Sadee W and Blower P: Chemoinformatics analysis identifies cytotoxic compounds susceptible to chemoresistance mediated by glutathione and cystine/glutamate transport system xc. J Med Chem 50: 1896-1906, 2007. 